Laure-Amélie LELOUVIER, Bruno BOSC-ZANARDO, Laurent BRUXELLES, Pierre CHALARD et Marc JARRY

\section{En Vignes, une halte de chasse tardiglaciaire à Marsan dans le Gers (France)}

\title{
Résumé
}

En amont d'un aménagement routier, une opération archéologique préventive a livré une petite occupation de plein air documentée par une industrie lithique bien préservée et datée. Ce site, découvert sur un versant de vallon, se caractérise par un corpus lithique peu étoffé (une centaine de pièces) mais suffisant pour aborder des notions telles que les stratégies de subsistance, les types de mobilités ou la gestion d'un territoire. En effet, l'identification pétrographique des matériaux exploités et la qualité des informations technologiques de cette série nous ont permis de reconstituer les chaînes opératoires avec un fractionnement manifeste dans le temps et dans l'espace. L'analyse typo-technologique a mis en évidence une production dominante de lamelles rectilignes obtenues par extraction sur tranche de support laminaire, supports eux-mêmes, peut-être, produits sur place et associés à quelques outils de fond commun. En dépit d'un taux de fragmentation élevé il a été possible de restituer, en partie, les chaines de production allouées à la réalisation d'une activité spécifique. Ainsi, le statut du site est évoqué à travers cette réalisation technique particulière, à savoir la réfection d'armatures de chasse (la fabrication et le remplacement des pointes de projectile) dans le cadre d'une halte de courte durée. L'identification de cette activité spécialisée et la gestion des matières premières exploitées dans ce cadre nous permettent d'appréhender des notions de circulations des hommes et des équipements sur, semble-t-il, de vastes territoires. L'occupation peut raisonnablement être attribuée au Tardiglaciaire sur la base du mobilier et d'une date radiocarbone. Cette production lamellaire témoignerait d'une permanence dans l'élaboration de l'équipement technique lié à la chasse. Comme il est fréquent pour ce type d'usage, la standardisation des produits et leur très faible diversité participent à un fond commun qui a pu perdurer jusqu'à la fin du Pléistocène, voire au-delà. Ce site est un des rares exemples dans cette région (le Gers) à témoigner d'un investissement ou réinvestissement d'un territoire et constitue à l'heure actuelle une donnée inédite pour cette période.

\section{Mots-clés}

Technologie lithique, armatures, halte de chasse, territoire, datation, Tardiglaciaire, Gers.

\footnotetext{
Abstract

Rescue excavation in advance of road construction uncovered a small open air site with a well preserved and well dated lithic industry. On the slope of a small valley, the site is characterized by a lithic industry which, although small in quantity (one hundred artefacts), provides good-quality technological information, enabling issues such as subsistence strategy,
} 
mobility and territories to be addressed. Typo-technological analysis revealed a production of rectilinear bladelets obtained from the edges of blade blanks, which were perhaps themselves produced on the site, as well commonly occurring tools. In spite of considerable fragmentation, it was possible to reconstruct part of the manufacturing process of backed-bladelets used for hunting activities. Thus the status of the site is reflected by this particular technical activity, involving the production of hunting weapons (the manufacture and replacement of projectile points) during short stays on the site. The identification of this specialized activity, related to acquisition of specific raw materials, provides a basis for discussing notions of circulation of people and equipment over apparently vast territories. The occupation can be attributed to the Late Glacial, on the grounds of the finds and one radiocarbon date. This bladelet manufacture is evidence for longlasting production of equipment linked to hunting. As is frequently the case for this kind of use, the standardization of products and their low diversity results in frequently occurring types that continue to be made until the end of the Pleistocene, and perhaps even later. This site is one of the few examples in the Gers region with evidence for occupation or reoccupation of a territory and provides new information for this period.

\section{Keywords}

Lithic technology, arrowheads, hunting camp, territory, dating, Lateglacial, Gers.

Au-delà de l'analyse typo-technologique des productions lithiques durant le Paléolithique, les problématiques actuelles sont davantage orientées vers les notions de territoires et de mobilités, comme le suggère un certain nombre de manifestations (Jarry et al., sous presse; Leduc et al., 2000; Jaubert et Barbaza, 2005; Bressy et al., 2006). En effet, le développement méthodologique des approches techno-fonctionnelles et environnementales (taphonomie, pétrographie, technoéconomie, géomorphologie, scléro-chronologie) permet de mieux exploiter la documentation dont nous disposons et de renseigner plus précisément les questions relatives aux stratégies de subsistance, aux registres d'activités d'un site ou aux types de mobilités intégrées dans la gestion d'un territoire.

Dans ce cadre, le site d'En Vignes, documenté par une industrie lithique bien préservée et datée, représente un jalon intéressant pour aborder certaines de ces problématiques. En effet, le caractère inédit de cette découverte régionale préfigure, sans aucun doute, de nouvelles approches concernant l'exploitation du territoire. La présentation de ce gisement a pour but de contribuer à une meilleure perception des activités concernant les groupes du Tardiglaciaire. Bien que ce site ne constitue qu'une courte séquence dans un système socio-économique plus vaste, cette découverte est toutefois suffisamment rare pour souligner l'intérêt qu'elle suscite.

\section{PRÉSENTATION}

Le site (fig. 1) a été mis au jour à l'issue d'une opération de diagnostic archéologique en amont d'un aménagement routier (Chopin, 2006). La présence singulière de galets de quartzite dans un niveau argilo- limoneux a révélé l'existence d'un niveau d'occupation plus important à l'origine d'une intervention de fouille réalisée par une équipe conjointe de l'INRAP et l'université Toulouse - Le Mirail.

Les choix méthodologiques ont été dictés par différents facteurs auxquels nous sommes habituellement confrontés dans le cadre des travaux d'archéologie préventive. En effet, il a fallu concilier la nécessité d'acquérir un maximum d'informations concernant la formation du niveau archéologique, la surface du gisement et son contenu (l'industrie lithique en plan et en altimétrie) dans un temps relativement court. Toutefois, la procédure est restée classique avec un décapage manuel du niveau archéologique à l'intérieur d'un carroyage métrique orthonormé associé à un tamisage à l'eau des sédiments afin de récupérer les plus petits éléments.

L'échantillon, restreint, a été récolté sur une surface limitée et dans un niveau unique de faible amplitude. Il est constitué pour l'essentiel de produits en silex et quelques pièces en quartzite.

Si nous pouvons regretter l'absence de restes fauniques identifiables, nous avons toutefois pu réaliser une date radiocarbone par AMS sur un fragment osseux préservé.

Les éléments recueillis autorisent une analyse technotypologique fine du matériel et offrent même l'opportunité d'appréhender des activités à travers des comportements techniques.

\section{CADRE ENVIRONNEMENTAL ET GÉOLOGIQUE}

Située aux confins de l'Astarac et du Haut-Armagnac, la région d'Auch rassemble les eaux provenant de 


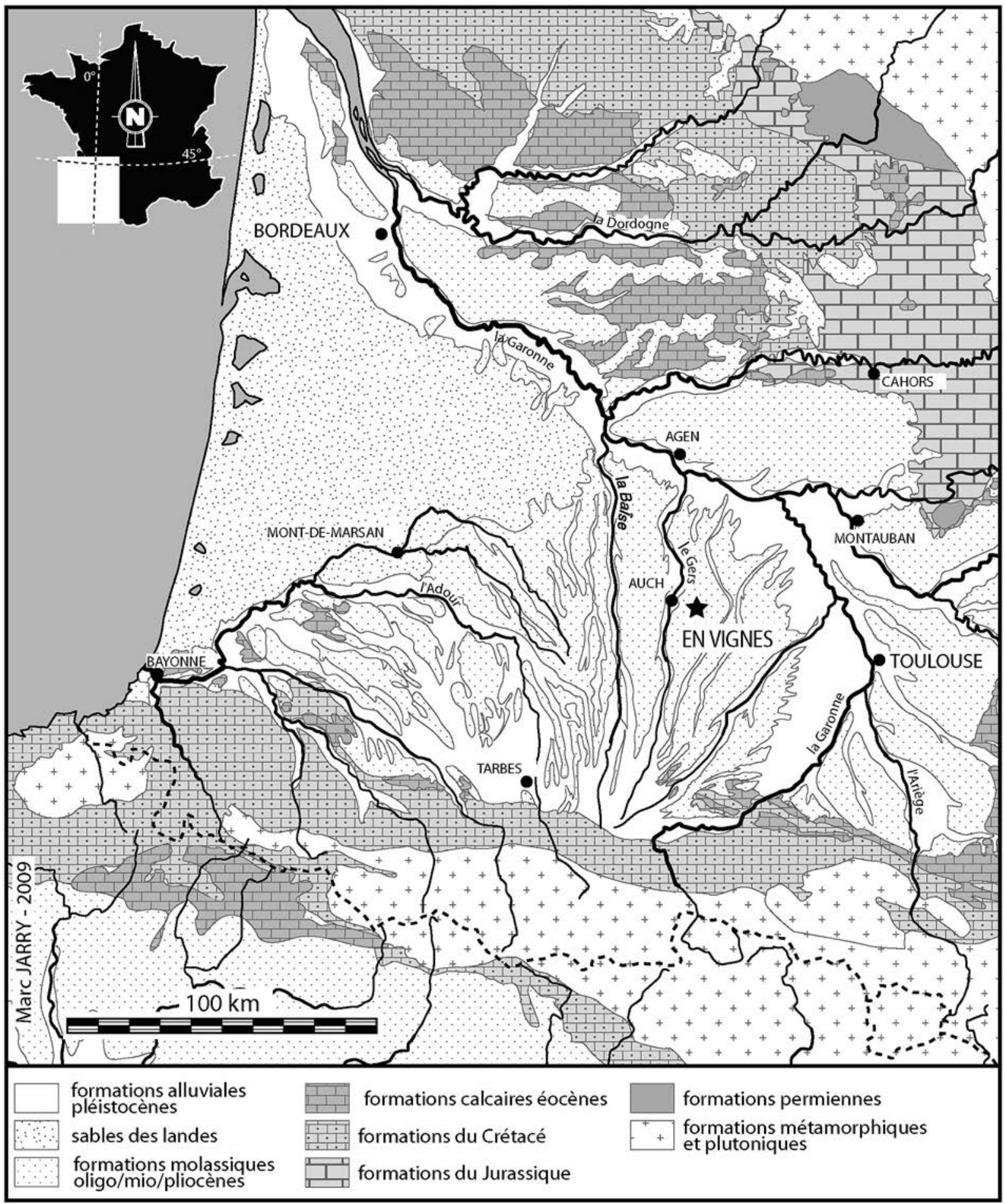

Fig. 1 - Localisation du site d'En Vignes à Marsan, Gers (DAO M. Jarry).

Fig. 1 - Location of the En Vignes site, Marsan, Gers (CAD M. Jarry).

nombreuses rivières en éventail (fig. 2) originaires des Pyrénées. D'est en ouest, on trouve l'Arrats, l'Arçon (dont est tributaire le vallon qui démarre au niveau du site), le Gers dans lequel viennent se jeter le Cédon et le Sousson puis, à l'ouest d'Auch, l'Audoue et la Baïse.

À partir du Quaternaire, l'encaissement du réseau hydrographique dans ce secteur a démantelé l'aplanissement général et a provoqué une réorganisation du réseau hydrographique. Toute une série de vallées affluentes se sont mises en place par érosion régressive à partir des principaux axes constitués par les cours d'eau descendant des Pyrénées. C'est notamment le cas du ruisseau du Baqué en amont duquel est localisé le site, qui rejoint le ruisseau de Leboulin, puis celui de Larroussagnet et enfin le ruisseau de 


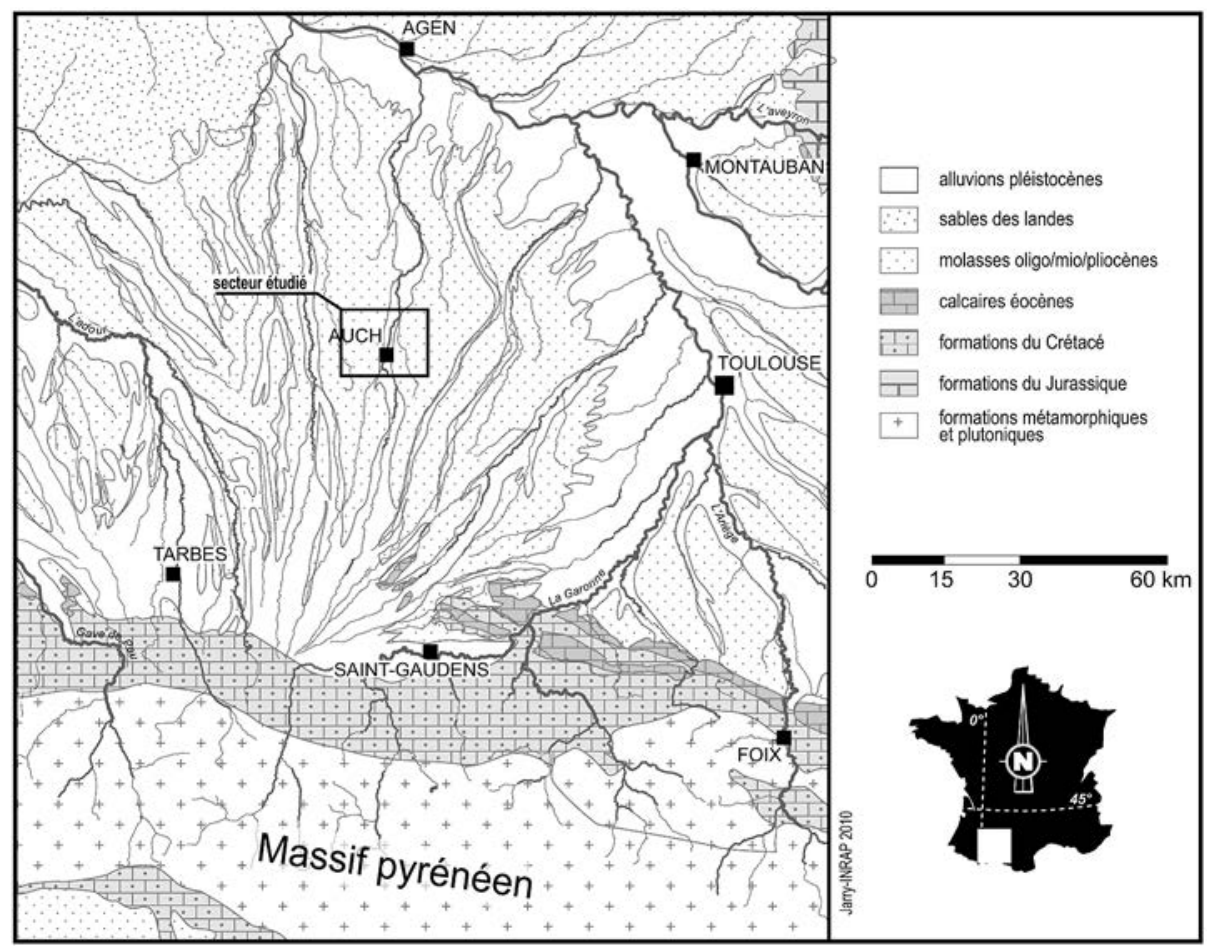

Fig. 2 - Cadre géologique de la région d'Auch d'après la carte géologique au 1/1000000 du BRGM, 2003 et Crouzel et al., 1973 (DAO L. Bruxelles et M.Jarry).

Fig. 2 - Geological setting of the Auch area, after the BRGM geological map 1:1,000,000, 2003 and Crouzel et al., 1973 (CAD L. Bruxelles and M.Jarry).

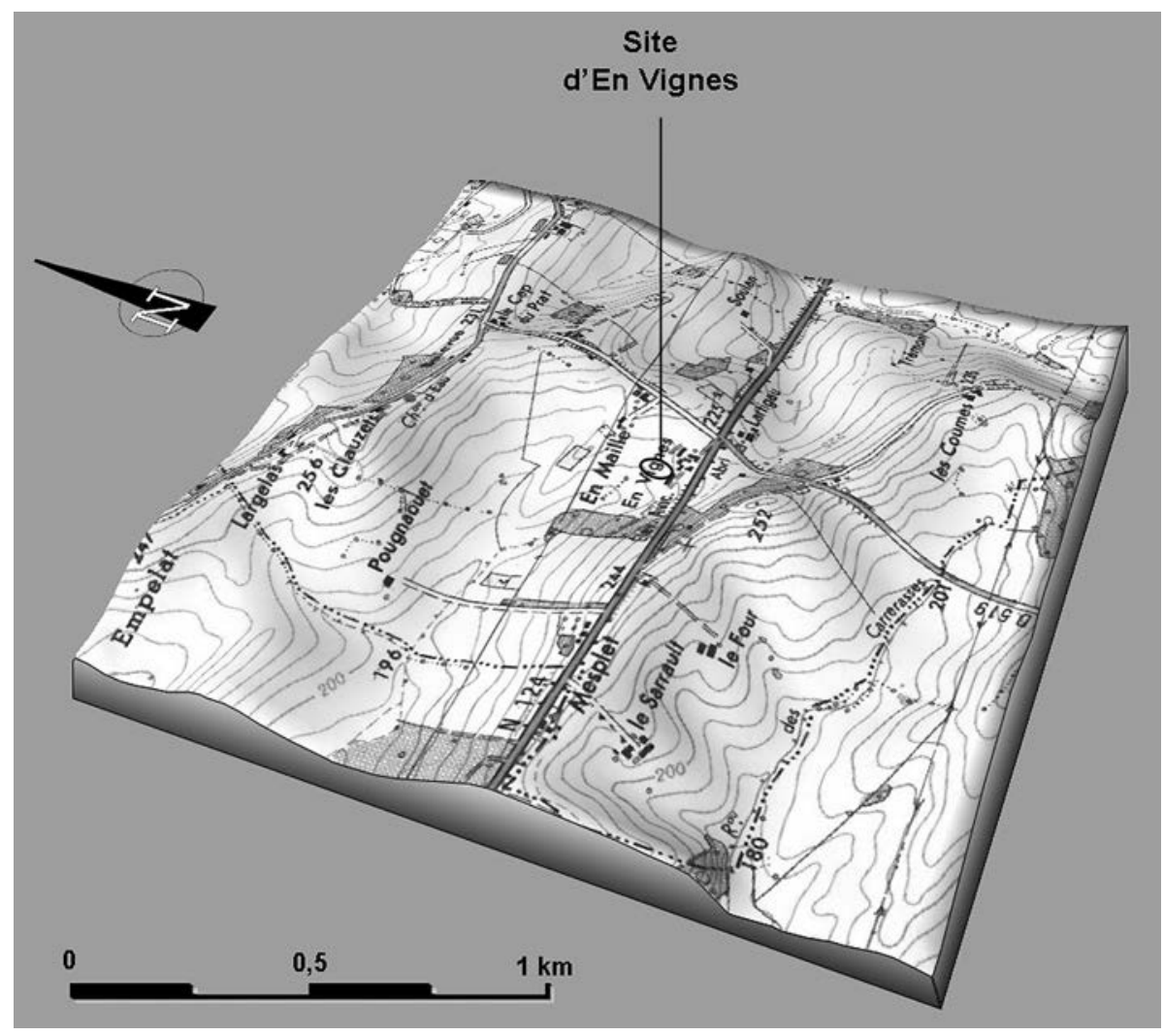

Fig. 3 - Bloc diagramme 3D illustrant le contexte topographique du site d'En Vignes (DAO L. Bruxelles). Fig. 3 - 3D block diagram illustrating the topographical context of the En Vignes site (CAD L. Bruxelles). 

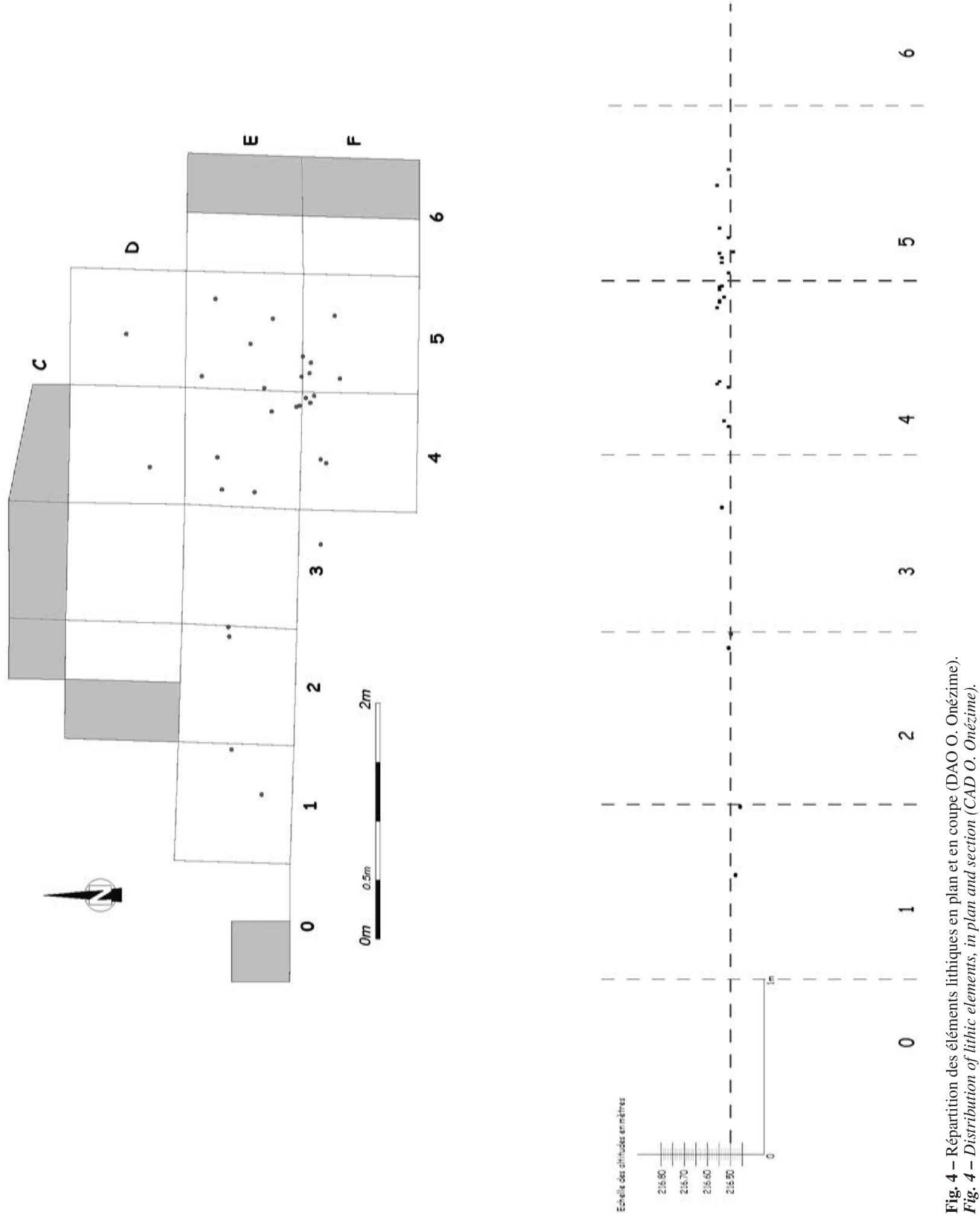
l'Arçon avant de se jeter dans le Gers. Ce réseau hydrographique, extrêmement hiérarchisé, isole de longues échines molassiques qui caractérisent le paysage de coteaux de cette région.

Le site a été découvert dans l'extrême amont du ruisseau du Baqué (fig. 3). Il est localisé une centaine de mètres en aval de l'interfluve séparant ce ruisseau de celui de Lama. À ce niveau, le vallon présente un fond large à peu près plat, inscrit d'une trentaine de mètres en contrebas du sommet des coteaux. Les écoulements de surface sont rares et ne se manifestent qu'après un épisode pluvieux. Un peu plus en aval, le fond du vallon, occupé par un écoulement plus ou moins pérenne, prend rapidement une forme en $\mathrm{V}$, ce qui traduit une dynamique érosive plus marquée qu'en amont.

L'industrie lithique découverte au cours de cette opération était prise dans une formation colluviale située en pied de versant. Le contexte topographique du site est relativement favorable à la préservation de ce type de vestiges. En effet, leur conservation est due essentiellement à la localisation en extrême amont du ruisseau du Baqué. Comme nous avons pu l'observer à plusieurs reprises dans ce type de contexte morphologique (Briand et al., 2005), seules les parties les plus amont du réseau hydrographique ont pu conserver des accumulations colluviales anciennes. À quelques dizaines de mètres plus en aval, les écoulements acquièrent très rapidement la compétence nécessaire pour recreuser ces formations meubles et donner à la vallée un profil en $\mathrm{V}$.

Les formations argilo-limoneuses dans lesquelles était contenu le matériel archéologique, correspondent à des dépôts de faible énergie. Ainsi, il apparaît que les écoulements responsables de la mise en place de cette accumulation colluviale avaient une compétence plutôt faible, due en particulier à l'absence d'un amont développé. Toutefois, il est difficile d'imaginer que ce site n'ait pas subi de perturbation, en particulier du fait de la nature meuble du substrat et des formations colluviales. Il semble, néanmoins, que la série n'a pas connu de remaniements successifs importants et que le matériel, prélevé un peu plus haut sur le versant, s'est redéposé à son pied, dès que la pente a diminué. Afin de quantifier ce phénomène, des analyses taphonomiques ont été réalisées sur ce matériel (composition granulométrique, répartition spatiale, état de surface des pièces archéologiques).

\section{ÉLÉMENTS DE TAPHONOMIE}

En préalable à l'analyse de l'industrie lithique, il convient d'appréhender les processus de formation du niveau archéologique et son contexte taphonomique.

La nappe de vestige se situe dans un niveau de faible épaisseur et s'étend sur une surface d'environ $15 \mathrm{~m}^{2}$ en suivant une pente douce vers l'ouest/nord-ouest où l'épandage va en s'amenuisant. Plusieurs arguments plaident en faveur d'une série homogène et représentative. En effet, une certaine concentration des vestiges a pu être observée sur $4 \mathrm{~m}^{2}$ et la dilatation verticale n'excède pas $8 \mathrm{~cm}$ (fig. 4). Nous verrons plus loin que même faible numériquement, l'ensemble lithique dévoile une grande cohérence technologique. Cependant, nous ne devons pas occulter les éventuelles altérations postdépositionnelles qu'un ensemble, à l'apparence homogène, a pu subir lors de son long séjour enfoui.

Les analyses géoarchéologiques récentes ont mis en évidence différents types de perturbations naturelles à l'origine de la mise en place des niveaux archéologiques tels que nous les retrouvons (Lenoble, 2003; Bertran et Lenoble, 2002). Ces déplacements peuvent être perçus à travers différentes approches conjuguées comme les tris granulométriques des objets, leurs dispositions en plan, leurs fabriques (pendage préférentiel) et les états de surface. L'analyse de ces derniers met en évidence une patine blanche (cacholong) homogène sur la totalité des pièces, à l'exception des quartzites.

Un déplacement horizontal de faible ampleur est perceptible à travers une disposition en éventail des objets lithiques suivant la pente naturelle et l'analyse granulométrique réalisée en fonction des classes de largeur des pièces montre une fraction fine bien représentée.

Le site a donc bien subi des perturbations postdépositionnelles mais le déplacement de faible ampleur ne semble pas avoir affecté de manière notable la série lithique dont l'intégrité et l'homogénéité technologique paraissent dans son ensemble assurée.

\section{L'INDUSTRIE LITHIQUE}

L'échantillon comprend cent quarante-deux pièces lithiques (tabl. 1). Bien qu'apparemment restreinte, la qualité des informations recueillies nous a permis, à travers cette série, de reconstituer les chaînes opératoires d'une production lamellaire.

\section{L'origine des matériaux}

Les silex exploités, par observation sous loupe binoculaire, ont été identifiés comme un silex blond d'origine

\begin{tabular}{|c|c|c|c|}
\hline & & \\
\hline & & Effectif & $\%$ \\
\hline \multicolumn{2}{|c|}{$\begin{array}{l}\text { Éclat laminaire } \\
\text { (+ retouches d'utilisation) }\end{array}$} & 1 & 0,70 \\
\hline \multicolumn{2}{|c|}{ Éclat lamellaire } & 1 & 0,70 \\
\hline \multicolumn{2}{|c|}{ Nucléus sur tranche } & 2 & 1,40 \\
\hline \multicolumn{2}{|c|}{ Lame (fragment proximal) } & 1 & 0,70 \\
\hline \multicolumn{2}{|c|}{ Éclats } & 12 & 8,45 \\
\hline \multicolumn{2}{|l|}{ Micro-éclats } & 18 & 12,67 \\
\hline \multicolumn{2}{|c|}{ Micro-esquilles } & 35 & 24,64 \\
\hline \multicolumn{2}{|c|}{ Débris } & 10 & 7,042 \\
\hline \multicolumn{2}{|c|}{$\begin{array}{l}\text { Lamelles brutes } \\
\text { (+ chutes de burin) }\end{array}$} & 30 & 21,12 \\
\hline \multirow[t]{2}{*}{ Outils dont } & \begin{tabular}{|l|} 
Burins/lame \\
\end{tabular} & 2 & 1,40 \\
\hline & Lamelles à dos & 27 & 19,01 \\
\hline \multicolumn{2}{|c|}{ Galet en quartzite } & 3 & 2,11 \\
\hline \multicolumn{2}{|c|}{ Total } & 142 & 100 \\
\hline
\end{tabular}

Tabl. 1 - Décompte de la série.

Table 1 - Composition of the series. 
marine totalement altéré. Il convient de rester prudent quant à sa provenance. Une seule pièce représentée par une armature a pu être identifiée plus précisément comme un silex du Flysch pyrénéen sensu lato (avec de nombreux fragments de bioclastes dont des spicules avec des inclusions orangées ou rouges). Il pourrait rentrer dans la variabilité décrite pour le silex du Flysch de Montgaillard (Barragué et al., 2001). L'ensemble des silex semble d'origine assez lointaine. En effet, jusqu'à une date très récente, les silicifications locales connues dans le Gers correspondaient à des formations tertiaires lacustres (secteur de Courrensan : Lacombe, 1998, p. 248). Cependant, des rognons de silex marins ont été découverts dans le nord-ouest du département lors d'un diagnostic archéologique réalisé en 2010 (Colonge et al., 2010). Les premières observations des échantillons prélevés conduisent à penser que ces silex sont vraisemblablement d'âge maestrichtien. De plus amples analyses seront nécessaires pour comparer la série d'En Vignes avec les matériaux siliceux mis au jour plus au nord (des travaux de caractérisation sont actuellement en cours par M. Bilotte et P. Chalard)

Les quelques éléments en quartzite gris bleuté sont issus, sans aucun doute, de la chaîne axiale des Pyrénées et ont pu être prélevés sous forme de galets présents dans les formations alluviales du Gers ou de l'Arrats, affluents de la Garonne. Ainsi, ces matériaux ont été récoltés dans un environnement plus proche que les silex.

\section{Une production lamellaire dominante pour des supports d'armatures}

L'examen des produits en silex a mis en évidence une production dominante de lamelles rectilignes obtenues par extraction sur tranche de support laminaire. La finalité concerne la fabrication de lamelles à dos.

\section{Les produits bruts}

Les produits allongés regroupent les sous-produits et les supports, a priori, de première intention (fig. 5). L'examen des lamelles révèle une fragmentation importante puisqu'une seule pièce est entière. La distribution des parties fragmentées est plutôt équilibrée avec seulement un léger déficit des parties distales, confirmant la bonne intégrité de la série. Ainsi, s'il est difficile de restituer la longueur recherchée par les tailleurs, les dimensions moyennes des largeurs et épaisseurs laissent apparaître une grande standardisation avec une largeur moyenne de 3,14 $\pm 1,42 \mathrm{~mm}$ et une épaisseur moyenne de 1,45 $\pm 0,59 \mathrm{~mm}$ (fig. 6). Les écart-types montrent des valeurs faibles. Nous avons noté que les normes morphométriques des lamelles retouchées ne correspondent pas exactement à celles observées sur les lamelles brutes présumant qu'elles ne sont pas issues de la production décrite.

Les talons sont quasiment tous punctiformes, liés à l'étroitesse du plan de frappe et à la technique de percussion utilisée (probablement la percussion directe au percuteur tendre organique ou minéral).
Parmi les produits d'entretien, les crêtes, les néocrêtes et les crêtes partielles sont assez nombreuses $(n=6)$. Elles se caractérisent par des micro-enlèvements sur la face dorsale, perpendiculaires à l'axe de débitage, parfois seulement présents dans la partie distale. Les produits lamellaires sont accompagnés de nombreux éclats et micro-éclats imputables à l'aménagement et à la réfection des plans de frappe. La série compte 30 éclats issus de ces phases d'entretien. Ils sont de dimensions relativement homogènes (fig. 7), en particulier concernant l'épaisseur, toujours constante (moyenne $=1,27 \pm 0,56 \mathrm{~mm}$ ). Une micro tablette de ravivage, dont un bord porte les ondulations de la face

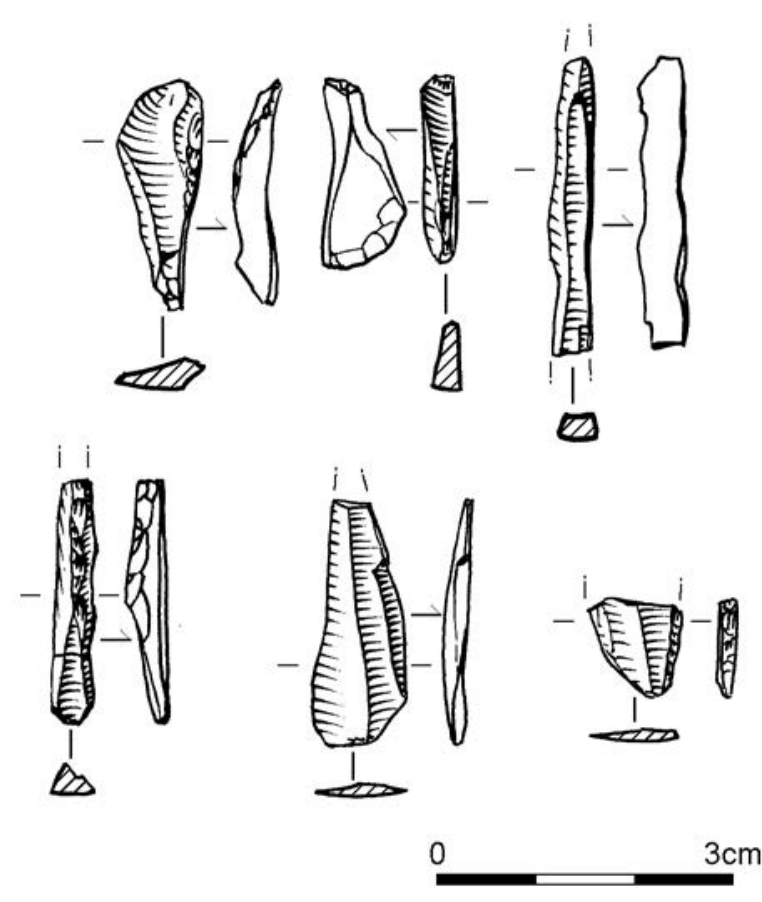

Fig. 5 - Les produits de débitage issus de la production lamellaire (dessin M. Jarry).

Fig. 5 - Debitage from bladelet production (drawing M. Jarry).

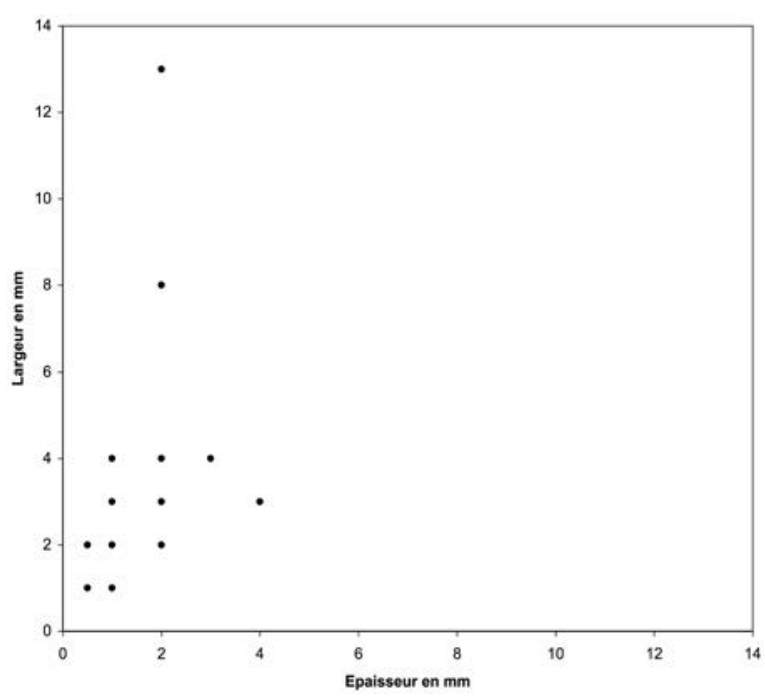

Fig. 6 - Module des lamelles brutes 1/e (en mm).

Fig. 6 - Raw bladelet module, width/thickness ( $\mathrm{mm}$ ). 


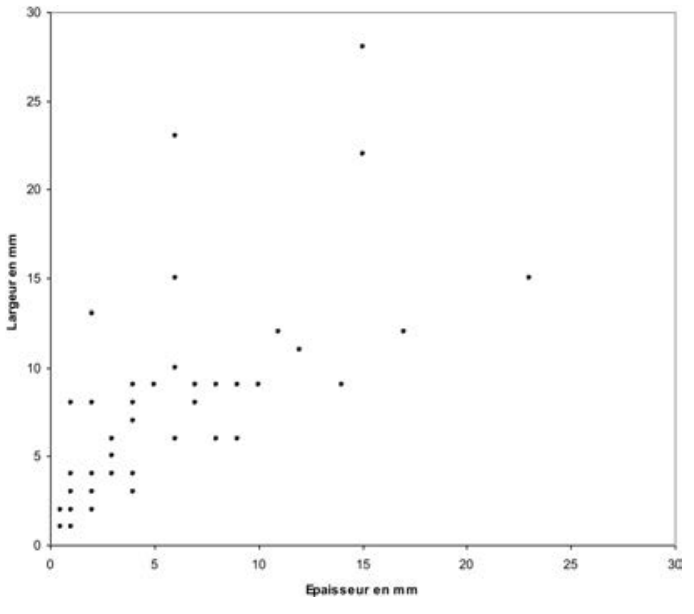

Fig. 7 - Module des éclats $1 / \mathrm{e}$ (en mm).

Fig. 7 - Flake module, width/thickness $(\mathrm{mm})$.

inférieure du nucléus, présente les négatifs des enlèvements antérieurs de lamelles sur son talon, attestant le renouvellement du plan de frappe.

Les supports bruts affichent un profil rectiligne. Ils sont peu épais avec parfois un pan naturel (type «chute de burin») ou à section symétrique à deux pans.

Ces caractéristiques, déjà observées par ailleurs (Langlais, 2004), sont générées par la mise en place d'un débitage sur tranche.

\section{Les modalités de débitage}

Nous sommes en présence de deux modalités avec une gestion du volume conçue différemment mais produisant des supports aux caractères morphotechniques analogues.

La première a consisté en l'installation d'une surface d'exploitation dans le sens longitudinal et poursuivie dans l'épaisseur du support. Le cintrage existe naturellement par l'intersection des deux surfaces du nucléus. Toutefois, différents procédés ponctuent cette exploitation par des séquences de réaménagement de la table et du plan de frappe. Ce dernier est entretenu par les enlèvements successifs de petits éclats ou bien par le retrait d'une tablette totale.

Les convexités distales ont été contrôlées par la réalisation de crêtes totales ou partielles. Cet entretien est nécessaire lors de l'exploitation d'une table rectiligne afin de diminuer les risques de réfléchissements en cas d'irrégularités de la carène distale (Langlais, 2004). Ces réfléchissements sont d'ailleurs visibles sur les derniers enlèvements du nucléus qui ont, peut-être, provoqué son abandon. Cette difficulté de produire des supports rectilignes est illustrée par un enlèvement de seconde génération, qui a largement outrepassé, emportant avec lui la table lamellaire.

De la même manière, l'enlèvement antérieur aux derniers négatifs réalisé sur la tranche mais dans le sens opposé pourrait correspondre à une volonté d'aplatissement de la carène, toujours dans l'optique d'une production rectiligne. En effet, les négatifs observés sur les lamelles brutes et les armatures montrent une orientation du débitage strictement unipolaire. Ainsi un des derniers enlèvements correspond à un éclat latéral réalisé aux dépens d'un plan de frappe opposé laissant entrevoir un possible recintrage de la surface lamellaire.

Le deuxième nucléus se distingue par l'orientation de sa table lamellaire installée transversalement par rapport à l'axe du support-nucléus.

Le détachement des produits allongés s'est fait aux dépens d'une surface lisse issue d'une fracture antérieure. Le contrôle de la convexité distale a été assuré par une crête encore largement visible sur le nucléus. Cette modalité consiste en une réduction progressive du nucléus; elle permet une production récurrente de lamelles dont la morphologie diffère des supports issus de l'autre nucléus. En effet, quelques lamelles brutes, souvent accompagnées d'enlèvements latéraux (restes de préparation de crête), présentent un profil courbe et une surface d'éclatement plus large. Toutefois, la progression du débitage devait conduire à une production de lamelles plus minces et rectilignes au regard des

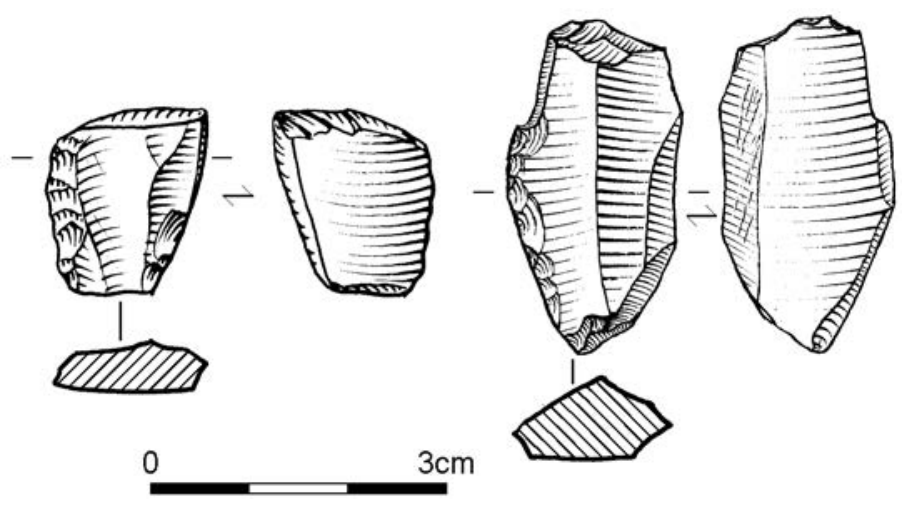

Fig. 8 - Les nucléus sur fragments de lame (dessin M. Jarry). Fig. 8 - Cores on blade fragments (drawing M. Jarry).

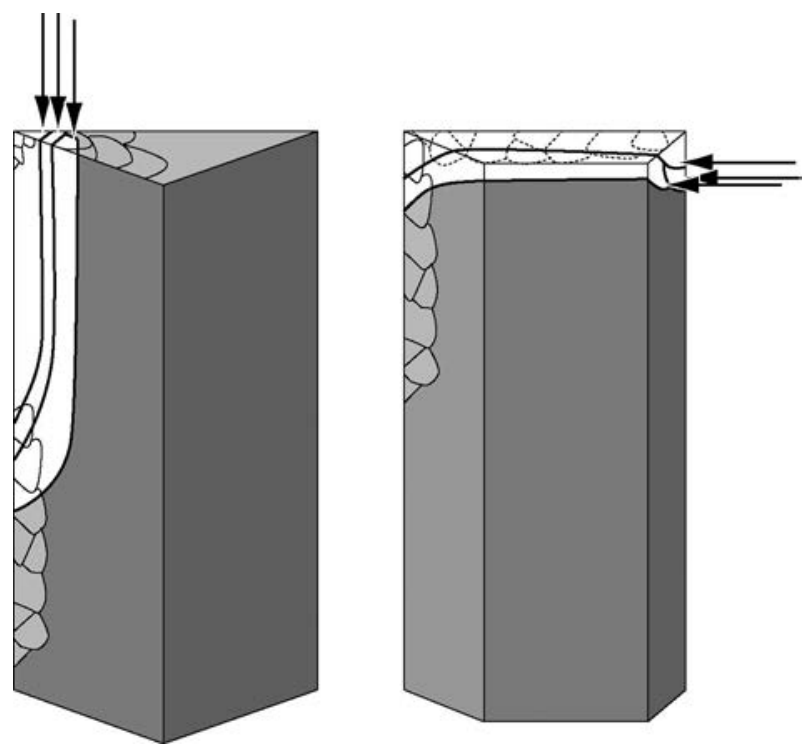

Fig. 9 - Schéma théorique des deux modalités du débitage lamellaire sur tranche (dessin M. Jarry).

Fig. 9 - Conceptual design of two forms of bladelet knapping from the edge of a flake (drawing M. Jarry). 
quelques supports à deux pans qui correspondent, certainement, à la largeur de la table. Il est de toute manière clair que la phase de transformation réalisée au moyen de la retouche abrupte a aussi contribué à réduire la largeur de ces pièces et ainsi retrouver les normes morphométriques recherchées (fig. 8 et fig. 9).

\section{Les lamelles à dos}

La série lithique comprend 55 lamelles sur un total de 136 pièces, soit 40,4\% de la série, avec 24 lamelles retouchées ${ }^{1}$, soit $17,6 \%$ du corpus. Toutes les lamelles retouchées sont à dos et fracturées (fig. 10).
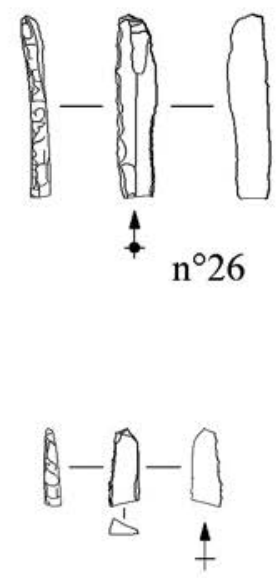

$\mathrm{n}^{\circ} 7$

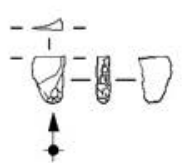

$n^{\circ} 84$

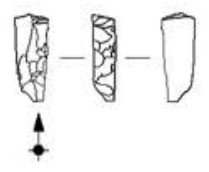

$\mathrm{n}^{\circ} 94$
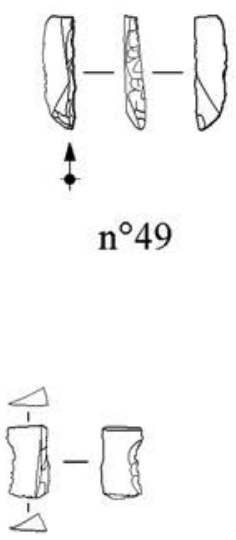

$n^{\circ} 8$

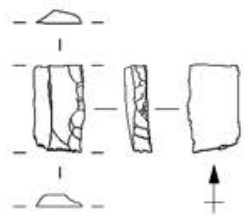

$n^{\circ} 91$
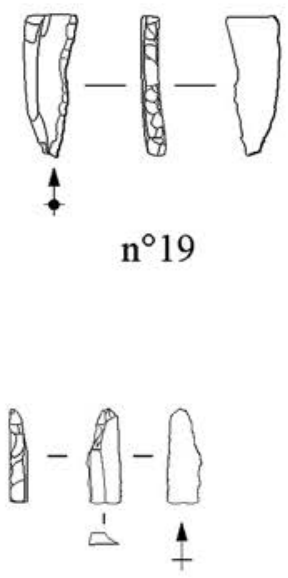

$n^{\circ} 108$

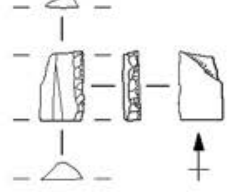

$\mathrm{n}^{\circ} 92$
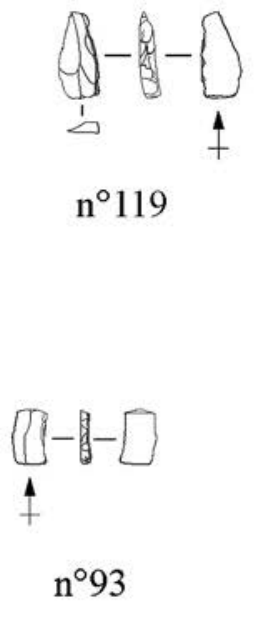

$\mathrm{n}^{\circ} 119$

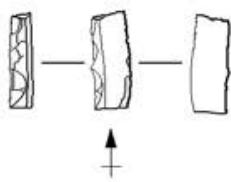

$\mathrm{n}^{\circ} 59$

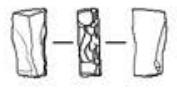

$\mathrm{n}^{\circ} 18$

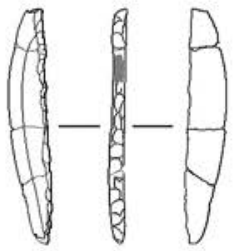

remontage

$102 / 10 / 104 / 105$

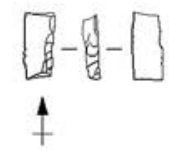

$\mathrm{n}^{\circ} 123$

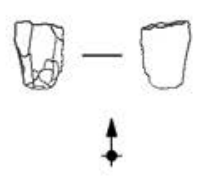

$\mathrm{n}^{\circ} 132$

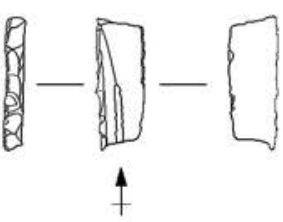

$\mathrm{n}^{\circ} 31$

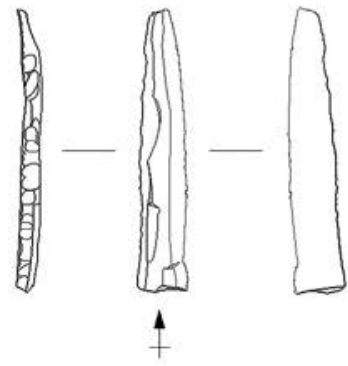

$\mathrm{n}^{\circ} 11$

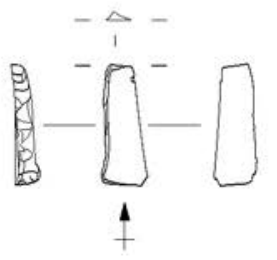

$n^{\circ} 96$

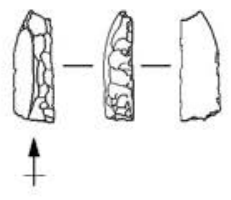

$\mathrm{n}^{\circ} 130$
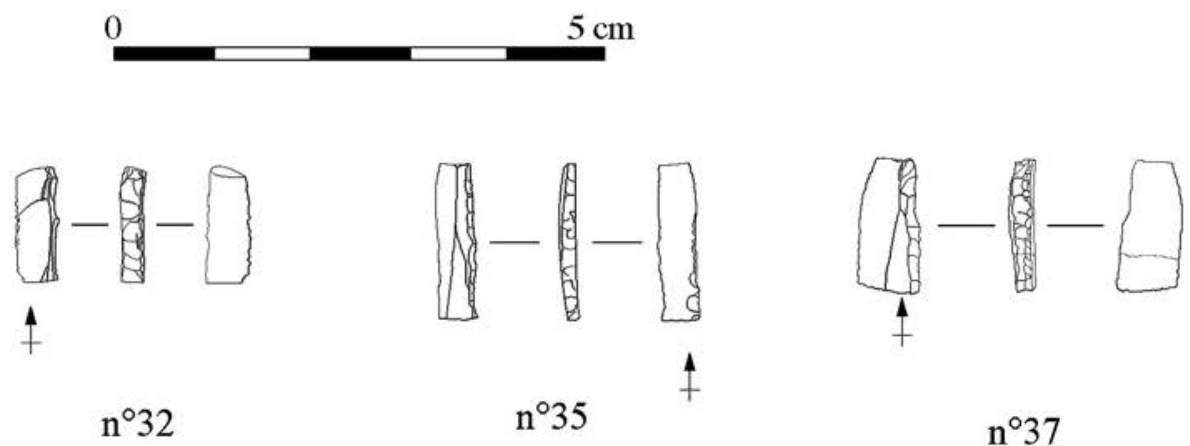

Fig. 10 - Les lamelles à dos (dessin B. Bosc-Zénardo).

Fig. 10 - Backed bladelets (drawing B. Bosc-Zénardo). 
Cet ensemble est très homogène d'un point de vue techno-typologique.

Concernant les caractères morphométriques, la longueur de ces pièces ne rend pas compte des dimensions initiales des supports ou des outils entiers. Les mesures prises ne sont mentionnées qu'à titre indicatif. Seuls deux exemplaires sont presque complets : ils affichent respectivement une longueur de $29 \mathrm{~mm}$ et de $24 \mathrm{~mm}$. La moyenne des longueurs des fragments est de $9,2 \mathrm{~mm}$ avec une nette concentration des pièces entre $7 \mathrm{~mm}$ et $11,5 \mathrm{~mm}$.

Il est plus intéressant d'aborder l'aplatissement. Il témoigne d'une très forte stabilité dimensionnelle. La grande majorité des individus a une largeur comprise entre 3 et 4 mm (fig. 11) et une épaisseur de 1 à 2 mm (fig. 12).

Ces lamelles à dos sont donc très standardisées. Les épaisseurs sont contrôlées lors de la production des supports et les largeurs par la mise en place d'un bord abattu.

La phase de retouche se caractérise par un dos bien marqué au moyen d'une retouche directe abrupte «normale» (Tixier, 1963), plus exceptionnellement semiabrupte (fig. 10). Sur de nombreux dos apparaît une reprise sous forme de micro-retouches (fig. 10, $\mathrm{n}^{\text {os }} 31$, 32 et 37). Celles-ci pourraient être une solution technique utilisée pour abraser les corniches résiduelles des premières lignes de retouches. Un seul exemplaire de lamelle à dos tronquée oblique (fig. 10, $\left.\mathrm{n}^{\circ} 108\right) \mathrm{a}$ pu être reconnu dans le corpus. Cependant, le déficit des

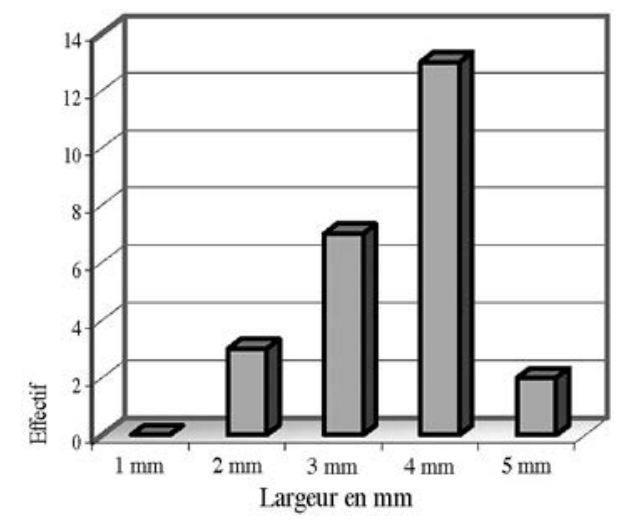

Fig. 11 - Distribution des individus selon leur largeur. Fig. 11 - Distribution of individuals according to width.

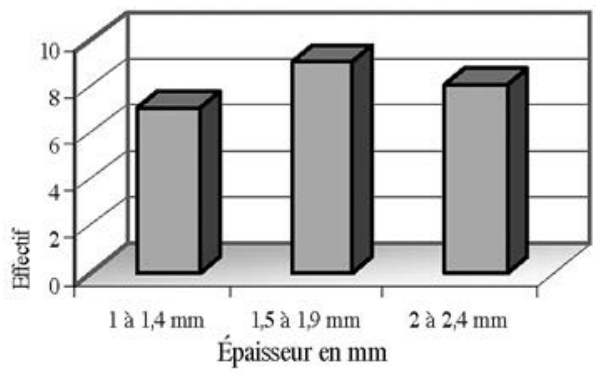

Fig. 12 - Distribution des individus selon leur épaisseur. Fig. 12 - Distribution of individuals according to thickness. parties apicales a pu nous amener à sous-estimer ce type d'armature.

La majorité des lamelles a un bord opposé au dos vif sans aménagement, excepté quatre pièces. Il s'agit, dans les deux cas, d'un léger grignotage imputable aux conditions taphonomiques (processus postdépositionnels, altération de la matière,...), contrairement à deux pièces qui présentent des ébréchures d'utilisation sur le tranchant resté brut.

L'emplacement des dos se situe majoritairement à droite, hormis six individus latéralisés à gauche. Deux pièces n'ont pu être orientées. Les lamelles sont trop peu nombreuses pour confirmer catégoriquement une latéralisation préférentielle des dos.

L'examen des talons révèle une très nette domination des punctiformes. Un exemplaire pourrait avoir un talon facetté. Les dimensions très réduites des supports, la faiblesse des épaisseurs notamment, expliquent la prédominance de ce type de talon.

\section{La fracturation des lamelles}

Les fragments représentés sont pour l'essentiel des parties proximales et mésiales. Ces cassures sont de quatre types : cassure nette (le bord présente une surface lisse sans stigmate), cassure par flexion (avec le rebroussé caractéristique), cassure burinante (trace d'un enlèvement en coup de burin), et un type de fracture que nous avons nommé «patinée altérée» (la surface du bord fracturé est très patinée et a un aspect bouchardé provoqué par l'altération du silex). Ce dernier type de fracture est le plus fréquent. Malheureusement cet aspect, lié à la désilicification et à l'altération du silex, ne rend pas possible l'identification du type de fracture d'origine.

Parmi les fractures diagnostiques (fig. 13), ce sont les cassures franches qui dominent avec $50 \%$ de cassures par flexion suivies par les cassures nettes (30\%).

Une pièce porte les stigmates clairs d'une fracture en gond ou en plume, selon la nomenclature proposée par A. Fischer (Fischer et al., 1984). Ce type de fracture a souvent été observé lors d'une utilisation en projectile, mais elle peut aussi être provoquée lors d'un

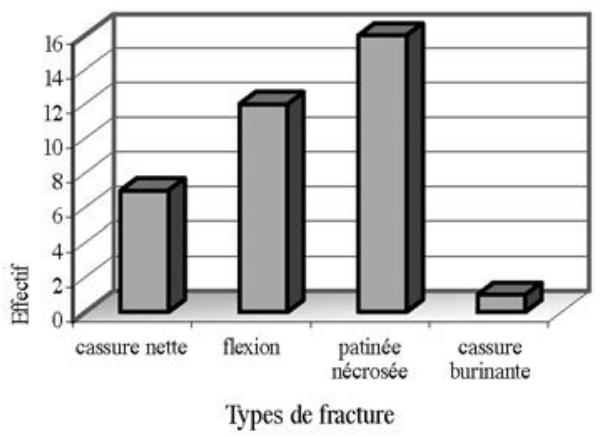

Fig. 13 - Distribution des lamelles selon le type de fracture. Fig. 13 - Distribution of bladelets according to type of fracture. 
accident de retouche ou d'un piétinement (Geneste et Plisson, 1986).

Il n'y a qu'une seule cassure burinante. La pièce $\mathrm{n}^{\mathrm{o}} 31$ (fig. 10) porte aussi des stigmates flagrants d'une fracture par flexion perpendiculaire à l'axe de débitage.

Le nombre important de fractures complexes (en flexion et en plume) associé à une bonne représentation des fragments proximaux et mésiaux plaide en faveur de l'utilisation de ces lamelles comme armatures (O'Farrel, 2004), et peut-être à l'origine du faible nombre de raccords «...dû à l'absence de certains éléments qui ont pu se perdre sur le lieu de chasse» (Cattin, 2002, p. 60).

\section{Réflexion sur les armatures}

L'analyse détaillée a souligné la cohérence de cette population de lamelles. Elles sont typo-métriquement très stables : une largeur comprise entre 3 et $4 \mathrm{~mm}$ et une épaisseur de 1 à $2 \mathrm{~mm}$. Les quelques talons encore conservés sont punctiformes. La majorité est latéralisée à droite et le dos est presque toujours obtenu par une retouche abrupte directe. Ces outils sont issus d'un véritable schéma normatif : production de supports aux épaisseurs standardisées et réalisation d'un dos par une retouche abrupte directe, le plus souvent sur le bord droit du support.

L'hypothèse la plus probable quant à l'utilisation de ces lamelles à dos est celle d'un emmanchement par ce même dos, le bord opposé non retouché servant de tranchant vulnérant. La littérature fournit certains exemples : découvertes archéologiques in situ, expérimentales (Allain et Descouts, 1957; Leroi-Gourhan, 1983; Bodu et Debout, 2009) ou exemples ethnographiques (Nuzhnyj, 1989).

Le fort taux de fracturation des pièces et le déficit des parties apicales ne permettent ni d'observer une standardisation des longueurs ni de préciser les catégories typologiques de ces lamelles à dos simples. Ces pièces se retrouvent en grand nombre dans certain faciès du Magdalénien moyen comme au Crès (Langlais, 2004) ou au Mas de Mayan (Breuil, 2001), mais associées généralement à des lamelles à dos tronquées.

\section{Une production en marge}

D'autres pièces suggèrent l'existence d'un débitage sur bloc de silex et galets de quartzite mais dont les modalités et les finalités sont plus difficiles à préciser.

Les produits bruts récoltés permettent d'envisager une autre méthode d'exploitation du silex, probablement à partir d'un petit bloc déjà préformé. Cette hypothèse est étayée par la présence de quelques éclats d'assez grandes dimensions, d'un éclat laminaire entier et la présence d'un fragment proximal de lame, semble-t-il, cassé au débitage et qui tranche avec les modules des nucléus lamellaires (fig. 14). Si les modalités ne peuvent être restituées à partir de ces éléments, nous pouvons, toutefois, envisager l'extraction de quelques lames dans le but d'obtenir des supports-nucléus dédiés à la production lamellaire. Le nucléus encore exploitable a pu être emmené par le groupe ou abandonné dans un secteur hors de la zone de fouille.

Par ailleurs, nous avons décompté deux burins «typiques», un burin sur cassure réalisé sur lame (fig. $15, \mathrm{n}^{\circ} 1$ ) et un burin dièdre déjeté sur fragment distal de lame (fig. 15, $\mathrm{n}^{\mathrm{o}} 2$ ).

Concernant le burin dièdre nous avons identifié un micro enlèvement situé sur le dièdre. Ce type de stigmate a fréquemment été observé sur des burins expérimentaux utilisés en rainurage sur bois animal (observation personnelle : L.-A. Lelouvier).

Ainsi, l'association burin-armature pour la fabrication ou le renouvellement de pointes de projectile peut constituer une proposition fonctionnelle solide.
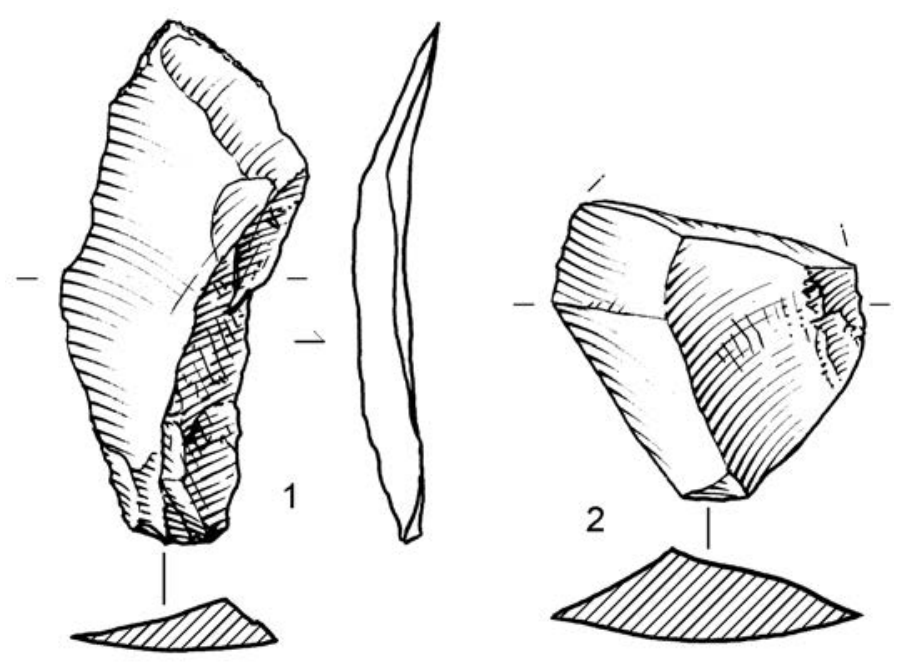

Fig. 14 - Les produits issus d'un autre mode de production (dessin M. Jarry).

Fig. 14 - Products resulting from a different mode of production (drawing M. Jarry).

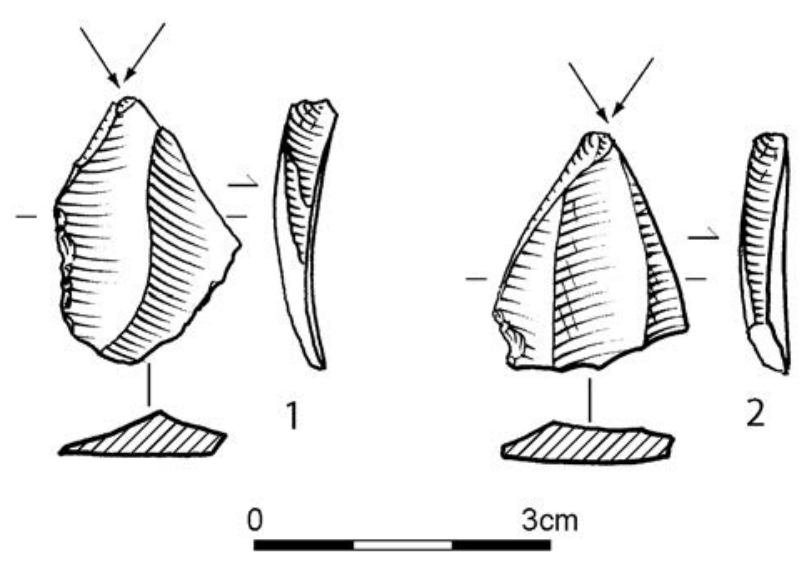

Fig. 15 - Les outils, 1 : burin sur cassure; 2 : burin dièdre (dessin M. Jarry).

Fig. 15 - Tools, 1: burin on break; 2: dihedral burin (drawing M. Jarry). 

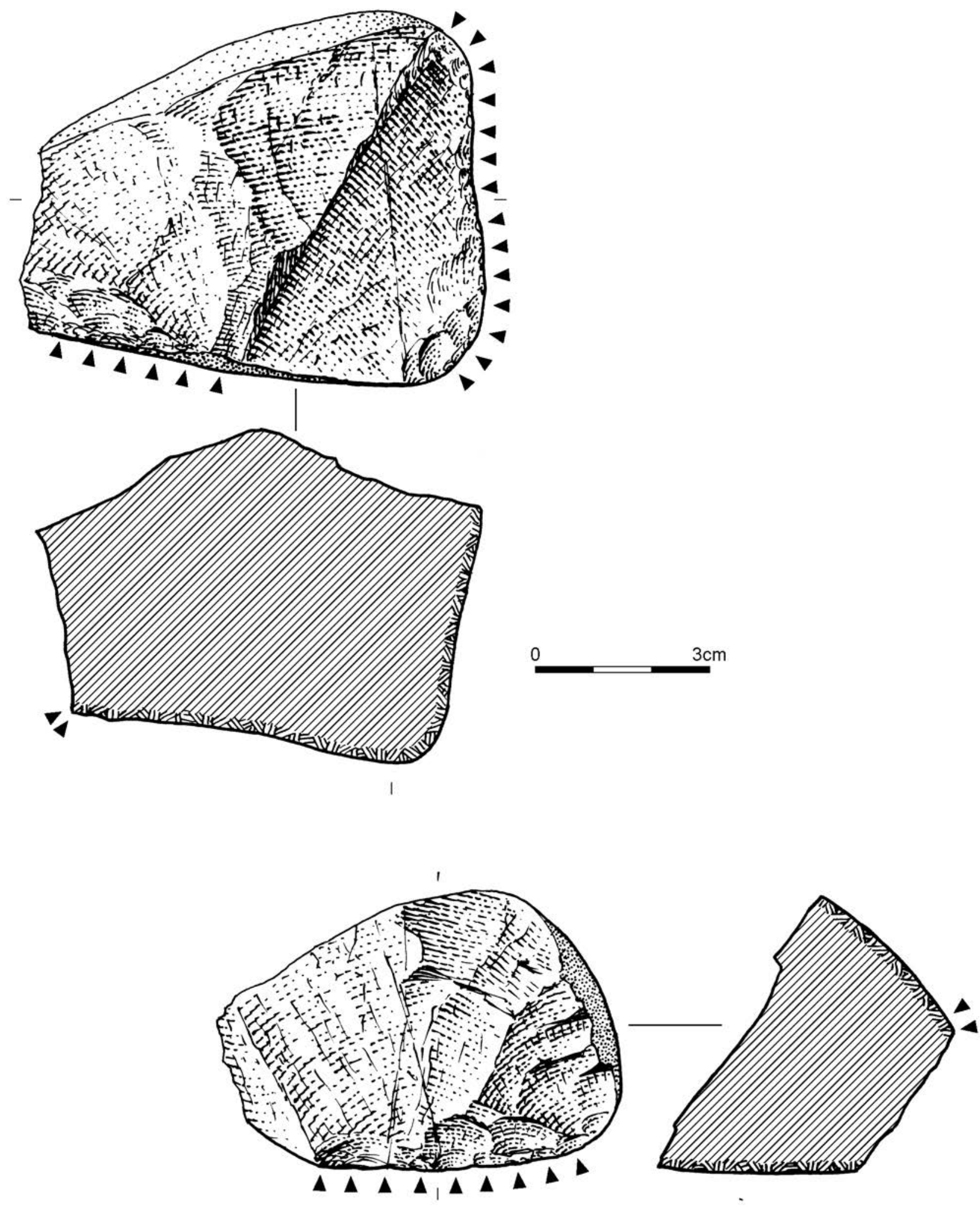

Fig. 16 - Les deux nucléus en quartzite associés à la production lamellaire (dessin M. Jarry). Fig. 16 - The two cores associated with quartzite bladelet production (drawing M. Jarry).

Enfin, deux galets de quartzite fragmentés présentant des enlèvements associés à des traces d'impacts légèrement martelées et deux petits éclats attestent d'une exploitation de ce matériau (fig. 16). L'un des deux peut être considéré comme un percuteur réemployé en nucléus avec trois négatifs d'enlèvements. L'autre galet présente un enlèvement perpendiculairement au plan de frappe, probablement débité par percussion sur enclume. Pour les deux, les éclats obtenus n'ont pas été retrouvés sur le site. 


\section{SYNTHĖSE ET INTERPRÉTATION}

\section{Une chaîne opératoire fractionnée}

Les informations technologiques mettent clairement en évidence une chaîne opératoire fractionnée dans le temps et dans l'espace. En effet, l'acquisition des matières premières semble s'être déroulée loin du lieu de production, peut-être vers les Pyrénées si nous considérons la pièce en silex du Flysch et ceux en silex marin, l'hypothèse d'un approvisionnement depuis le nord du Gers suite à de récentes découvertes (op. cit.) restant encore à valider. En l'état actuel, ces matériaux témoignent de circulation sur un territoire assez étendu. Concernant les premières phases (test, mise en forme des blocs) elles se sont déroulées en dehors de la zone fouillée. En fait, les éléments in situ témoignent de la seule phase de production sans les produits issus de cette production, qui ont été exportés puisque les lamelles à dos (armatures) retrouvées sur place appartenaient sans conteste à une autre chaîne de production. Ainsi nous sommes bien en présence d'une séquence opératoire que nous pouvons estimer brève au regard du nombre de pièces abandonnées, dans un lieu donné pour un objectif précis (le remplacement des armatures).

\section{Les données culturelles et chronologiques}

Les productions lamellaires se développent dès le début du Paléolithique supérieur. Des travaux récents ont mis en évidence différentes méthodes de production lamellaire sur support-nucléus. Il en ressort, néanmoins, que des choix et des traitements distincts concernant ces supports ont été observés selon les grandes périodes culturelles. Ainsi, les Aurignaciens ont préférentiellement utilisé des supports d'éclats épais (pièces carénées) et procédé à un débitage frontal (Bon, 2000 ; Bordes, 2002) : ce sont les nucléus de type «grattoir caréné». Les Gravettiens ont exploité de grandes lames qui deviennent morphologiquement proches des burins (Klaric, 2003). Au Magdalénien, les modalités de production lamellaire se diversifient avec la reconnaissance des lamelles type Rocher de La Caille (Alix et al. 1995), les lamelles d'Orville (Pélegrin, 1982; Perlès, 1983; Parisot, 1995; Chehmana, 2009), sur tranche d'éclat type «burin transversaux» (Bodu, 2003; Chehmana, 2004; Ducasse et Langlais 2008, Ducasse 2010) ou sur tranche de lame (Langlais, 2004 et 2007). Si l'analyse techno-typologique démontre clairement les activités de taille et les objectifs, l'absence d'outils spécifiques nous renseigne peu sur une attribution chronologique précise. Toutefois, les caractères morphotechniques des lamelles et la gestion du débitage nous orientaient initialement vers un Magdalénien indifférencié.

Deux fragments osseux prélevés lors de la fouille ont été envoyés pour datations radiocarbone par AMS. Un seul échantillon a fourni un résultat : 11419-10000 cal. BC ou $10800 \pm 250$ BP (AMS ETH-30797).
Cette date placerait l'industrie à l'Épipaléolithique. Faute de comparaisons régionales pertinentes, il nous est difficile d'apprécier avec certitude cette unique date. Elle témoignerait d'une permanence dans l'élaboration de l'équipement technique lié à la chasse.

Comme il est fréquent pour ce type d'activité, la standardisation des produits et leur très faible diversité participe à un fond commun qui a pu perdurer durant tout le Tardiglaciaire et au-delà (Amiel et Lelouvier, 2002).

\section{Une halte de chasseurs}

Le matériel renvoie, donc, à la fabrication d'armatures qui semble, dans ce cas précis, correspondre à la réfection de pointes de projectile (ou le remplacement des pointes de projectile). La petite production marginale d'éclats peut s'intégrer dans le schéma opératoire de la production lamellaire en fournissant les supportsnucléus ou pour une production mixte ou autonome dans le but de satisfaire à d'autres besoins. Il en est de même pour les quelques éclats en quartzite qui ont pu servir à un éventuel traitement des carcasses ou être liés à la maintenance de l'équipement. Ainsi, s'il est ambitieux d'interpréter cette petite occupation sans les restes fauniques pour discuter des notions de territoire, il n'en reste pas moins tentant de voir à travers ce site un bon exemple d'une halte de courte durée dans l'excursion d'un groupe de chasseur, peut-être d'origine lointaine, transportant avec eux les matériaux nécessaires au renouvellement rapide de l'équipement de chasse. Ainsi, ils pouvaient évoluer dans un territoire relativement vaste (compte tenu de l'origine des matériaux) tout en restant relié à un campement plus important. L'hypothèse d'une adaptation technique efficace dans le cadre d'une mobilité logistique a déjà été évoquée (Bon, 2005; Bordes et al., 2005).

L'isolement géographique du site et les données disponibles ne nous permettent pas d'aller au-delà pour l'instant.

\section{CONCLUSION}

L'ensemble des lamelles, associé à deux burins et un éclat laminaire à «retouches d'utilisation» renvoient sans conteste à une ou à des activités spécifiques où la réfection des armatures est essentielle. Ainsi, ce site est le vestige d'une occupation vraisemblablement de courte durée («halte»), liée à une activité spécialisée (chasse). Cette occupation s'inscrit dans un territoire assez vaste, au regard de l'origine de certaines matières premières. Le site d'En Vignes ne doit donc pas être considéré comme isolé et autonome, il doit être intégré à un système plus large de sites complémentaires dont nous ne percevons ici qu'un maillon.

Le Dernier Maximum Glaciaire dans le Bassin aquitain est marqué par le no man's land des Landes (Klingebiel et Legigan, 1985; Bertran et al., 2009; Bertran et al., à paraître) et la possible désertion de la vallée de la Garonne, séparant ses deux rives (Jarry, 
2010; Bruxelles et Jarry, 2011). L'amélioration climatique du Tardiglaciaire est probablement à l'origine d'une reconquête des espaces aussi bien dans les milieux montagnards (Demars, 2008) que dans les grands plaines. Aussi s'il est difficile de préciser qu'elle est la place d'En Vignes dans ce nouveau schéma d'occupation, il est indéniable qu'il en constitue un des rares chaînons que les sites de plein air s'efforcent de dévoiler peu à peu.

\section{NOTE}

(1) Le nombre total de fragments de lamelles à dos se porte à 27 individus, cependant quatre fragments se raccordent, ce qui réduit le total à 24 .

\section{RÉFÉRENCES BIBLIOGRAPHIQUES}

ALIX P., PELEGRIN J., DELOGE H. (1995) - Un débitage original de lamelles par pression au Magdalénien de Rocher de la Caille (Loire, France), Paléo, 7, p. 187-200.

ALLAIN J., DESCOUTS J. (1957) - À propos d'une baguette à rainure armée de silex découverte dans le Magdalénien de Saint-Marcel, L'Anthropologie (Paris), 61, 5-6, p. 503-512.

AMIEL C., LELOUVIER L.-A. (2002) - Gisements post-glaciaires en Bas-Quercy : variabilité des espaces et des statuts de deux occupations mésolithiques sauveterriennes de plein air, Toulouse, Archives d'écologie préhistorique, 2002 (Recherche en archéologie préventive 1), $203 \mathrm{p}$.

BARRAGUE J. et E., JARRY M., FOUCHER P., SIMONNET R (2001) - Le silex du Flysch de Montgaillard et son exploitation sur les ateliers du Paléolithique supérieur à Hibarette (Hautes-Pyrénées), Paléo, 13, p. 29-52.

BERTRAN P., LENOBLE A. (2002) - Fabriques des niveaux archéologiques : méthode et premier bilan des apports à l'étude taphonomique des sites paléolithiques, Paléo, 14, p. 13-28.

BERTRAN P., ALLENET G., GÉ T., NAUGHTON F., POIRIER P SANCHEZ-GONI M.-F. (2009) - Coversands and Pleistocene Paleosols in the Landes Region, Southwestern, France, Journal of Quaternary Science, 24, 3, p. 259-269.

BERTRAN P., BANK W. E., BATERMAN M. D., DEMARS P.-Y. LANGLAIS M., LENOIR M. (à paraître) - Les Landes de Gascogne désert périglaciaire et frontière culturelle au Paléolithique, in Des Climats et des Hommes : glaciologie, climatologie, archéologie, histoire, actes du colloque (Paris, 19-21 novembre 2009), Paris, INRAP, Météo-France et Cité de la Science.

BODU P., DEBOUT G. (2009) - Les lamelles à dos de type «Pincevent» : fabrication, comportement lors des tris et fractures d'utilisation, in B. Valentin (dir.), Paléolithique final et Mésolithique dans le Bassin parisien et ses marges. Habitats, sociétés et environnements, projet collectif de recherche, programme P7, P8 et P10, rapport d'activités 2009 , p. 35-40.

BODU P. (2003) - Le Badegoulien de la Nièvre est un Badegoulien à lamelles à dos! Nouvelles découvertes effectuées sur le site de Oisy dans la Nièvre, in P. Bodu et S. Soriano (coord.), Le Paléolithique supérieur ancien au Centre et au Sud du Bassin parisien. Des systèmes techniques aux comportements, rapport de PCR (programme 4), région Centre nord, 2003, p. 79-84.

BON F. (2000) - La question de l'unité technique et économique de l'Aurignacien : réflexions sur la variabilité des industries lithiques à partir de l'étude comparée de trois sites des Pyrénées françaises (La Tuto de Camalhot, Régismont-le-Haut et Brassempouy), thèse de doctorat, université Paris I - Panthéon-Sorbonne, Paris, 425 p.

BON F. (2005) - Little Big Tool. Enquête autour du succès de la lamelle. Productions lamellaires attribuées à l'Aurignacien : chaînes opératoires et perspectives technoculturelles, in XIV Congrès de l'UISPP (Liège 2-8 septembre 2001), Luxembourg, MNHA, p. 479484.

BORDES J.-G. (2002) - Les interstratifications Châtelperronien/ Aurignacien du Roc-de-Combe et du Piage (Lot, France). Analyse taphonomique des industries lithiques; implications archéologiques, thèse de doctorat, université Bordeaux I, Bordeaux, 365 p.
BORDES J-G., BON F, LE BRUN-RICALENS F (2005) - Le transport des matières premières lithiques à l'Aurignacien entre le Nord et le Sud de l'Aquitaine : faits attendus, faits nouveaux, in J. Jaubert et M. Barbaza (dir.), Territoires, déplacements, mobilités, échanges durant la Préhistoire, actes du $126^{\mathrm{e}}$ Congrès national des sociétés historiques et scientifiques (Toulouse, 2001), Paris, CTHS, p. 185198.

BRESSY C., BURKE A., CHALARD P., MARTIN P. (2006) - Notions de territoire et de mobilité : exemples de l'Europe et des premières notions en Amérique du Nord avant le contact européen, actes de sessions présentées au $\mathrm{X}^{\mathrm{e}}$ Congrès annuel de l'association européenne des archéologues (EAA) (Lyon 8-11 septembre 2004), Liège, Études et recherches archéologiques de l'université de Liège (ERAUL 116), $169 \mathrm{p}$.

BREUIL J.-Y. (2001) - Gisements du Paléolithique supérieur et de l'Épipaléolithique, document final de synthèse de diagnostic archéologique, AFAN, $87 \mathrm{p}$

BRGM (2003) - Carte géologique de la France à l'échelle du millionième, $6^{\mathrm{e}}$ édition révisée, Orléans, 6 p. et 2 cartes hors texte.

BRIAND J., BATS J.-C., BRUXELLES L., FILHOL C., GRANCHA A., ONEZIME O. (2005) - RN 88, section La Baraque Saint-Jean, La Mothe (Aveyron), rapport de diagnostic archéologique, INRAP Grand Sud-Ouest, Toulouse, $48 \mathrm{p}$.

BRUXELLES L., JARRY M. (2011) - Climatic Conditions, Settlement Patterns and Cultures in the Paleolithic : the Exemple of the Garonne Valley (Southern France), Journal of Human Evolution, 61, p. 538 548.

CATTIN M.-I. (2002) - Hauterive-Champréveyres, 13. Un campement magdalénien au bord du lac de Neuchâtel : exploitation du silex (secteur 1), Neuchâtel, Service et musée cantonal d'archéologie (Archéologie neuchâteloise, 26), 2 vol.

CHEHMANA L. (2004) - Enquête sur l'identité du Badegoulien et sur ses origines possibles. Apport de l'étude des industries lithiques badegouliennes de Oisy (Nièvre) et solutréennes de Saint-Sulpicede-Favière (Essonne), mémoire de DEA, université Paris I - PanthéonSorbonne, Paris, $45 \mathrm{p}$.

CHEHMANA L. (2009) - The Conception of "Bertonne" and "Orville" Bladelet Production (Upper Paleolithic, France), Human evolution, 24, 2, p. 131-139.

CHOPIN J.-F. (2006) - Mise en $2 \times 2$ voies de la RN 124 Auch-Aubiet (2004-2005), rapport de diagnostic et d'évaluations, INRAP Grand Sud-Ouest, 83 p.

COLONGE D., ARRIGHI V., BERTRAN P., CHALARD P. (2010) A Saint-Aubin (Midi-Pyrénées, Gers, Réans et Cazaubon), rapport final de diagnostic archéologique, INRAP Grand Sud-Ouest, SRA Midi-Pyrénées, 48 p.

CROUZEL F., KIEKEN M., PARIS J.-P. (1973) - Notice explicative de la carte géologique de France, Feuille d'Auch, $n^{\circ}$ 981, Orléans, BRGM, 12 p.

DEMARS P.-Y. (2008) - Paléogéographie des chasseurs de l'Europe du Paléolithique supérieur: répartition et spécialisation des sites, L'Anthropologie, 112, p. 157-167. 
DUCASSE S. (2010) - La «parenthèse» badegoulienne : fondements et statut d'une discordance industrielle au travers de l'analyse technoéconomique de plusieurs ensembles lithiques méridionaux du Dernier Maximum Glaciaire, thèse de doctorat, université Toulouse II Le Mirail, Toulouse, $413 \mathrm{p}$.

DUCASSE S., LANGLAIS M. (2008) - Interprétation technologique et discussion autour du statut culturel des «pièces de la Bertonne». L'exemple de la série lithique de Seyresse (Landes, France), Paléo, 20, p. 59-88.

FISCHER A., VEMMING-HANSEN P., RASMUSSEN P. (1984) Macro and Micro Wear on Lithic Projectile Points. Experimental Results and Prehistoric Examples, Journal of Danish Archaeology, 3 , p. $19-46$.

GENESTE J.-M., PLISSON H. (1986) - Le Solutréen de la grotte de la Combe Saunière 1 (Dordogne), première approche palethnologique, Gallia Préhistoire, 29, p. 9-27.

JARRY M. (2010) - Les groupes humains du Pléistocène moyen et supérieur en Midi toulousain : contextes, ressources et comportements entre Massif central et Pyrénées, thèse de doctorat, université de Toulouse II - Le Mirail, Toulouse, 470 p.

JARRY M., BRUGAL J.-P., FERRIER C. (sous presse) - Modalite d'occupation et exploitation des milieux au Paléolithique dans le Sud-Ouest de la France : l'exemple du Quercy, actes du colloque organisé dans le cadre du XV Congrès de l'UISPP (Lisbonne, septembre 2006), session C67, Les Eyzies-de-Tayac, SAMR (Supplément à Paléo 5).

JAUBERT J., BARBAZA M. (2005) - Territoires, déplacements, mobilité, échanges durant la Préhistoire. Terres et hommes du Sud, actes du $126^{\text {e }}$ Congrès national des sociétés historiques et scientifiques (Toulouse, 2001), Paris, CTHS, 560 p.

KLARIC L. (2003) - L'unicité technique des industries à burins du Raysse dans leur contexte diachronique : réflexions sur la diversité culturelle au Gravettien à partir des données de La Picardie, d'Arcysur-Cure, de Brassempouy et du Cirque de la Patrie, thèse de doctorat, université Paris I - Panthéon-Sorbonne, Paris, 426 p.

KLINGEBIEL A., LEGIGAN P. (1985) - Histoire géologique de la Grande Lande, in La Grande Lande: histoire naturelle et géographique, actes du colloque (Sabres 27-29 novembre 1981), Paris Saugnac - Muret, CNRS et Parc naturel des Landes de Gascogne, p. $13-30$.

LACOMBE S. (1998) - Stratégies d'approvisionnement en silex au Tardiglaciaire. L'exemple des Pyrénées centrales françaises, Bulletin de la Société préhistorique Ariège-Pyrénées, 53, p. 223-266.

LANGLAIS M. (2004) - Les lamelles à dos magdalénienne du Crès (Béziers, Hérault : variabilité des modalités opératoires et stabilité typométrique), Préhistoire du Sud-Ouest, 11, p. 2-38.

LANGLAIS M. (2007) - Dynamiques culturelles des sociétés magdaléniennes dans leurs cadres environnementaux. Enquête sur 7000 ans d'évolution de leurs industries entre Rhône et Èbre, thèse de doctorat, université Toulouse II - Le Mirail, Toulouse, et université de Barcelone, $550 \mathrm{p}$.

LEDUC M., VALDEYRON N., VAQUER J. (2000) - Sociétés et espaces. Rencontres méridionales de Préhistoire récente, $3^{\mathrm{e}}$ session (Toulouse, 6-7 novembre 1998), Toulouse, Archives d'écologie préhistorique, $462 \mathrm{p}$.

LENOBLE A. (2003) - Le rôle du ruissellement dans la formation des sites préhistoriques : approche expérimentale, thèse de doctorat, université Bordeaux 1, Bordeaux, 297 p.

LEROI-GOURHAN A. (1983) - Une tête de sagaie à armatures de lamelles de silex à Pincevent (Seine-et-Marne), Bulletin de la Société préhistorique française, 80,5 , p. 154-156.
NUZHNYJ D. (1989) - L'utilisation des microlithes géométriques et non géométriques comme armatures de projectiles, Bulletin de la Société préhistorique française, 86, 3, p. 88-96.

O'FARREL M. (2004) - Les pointes de la Gravette de Corbiac (Dordogne) et considérations sur la chasse au Paléolithique supérieur ancien, in P. Bodu et C. Constantin (dir.), Approches fonctionnelles en Préhistoire, actes du XXV Congrès préhistorique de France (Nanterre, 24-25 novembre 2000), Paris, Société préhistorique française (Congrès de la SPF), p. 121-138.

PARISOT O. (1995) - Analyse typo-technologique du matériel lithique du site d'Orville (Indre, France), mémoire de maîtrise, université Paris X - Nanterre, $145 \mathrm{p}$.

PELEGRIN J. (1982) - Approche expérimentale de la technique de production lamellaire d'Orville, Studia Praehistorica Belgica, 2. p. $149-158$.

PERLES C. (1983) - Une technique originale de débitage des lamelles dans le site d'Orville (Indre), Bulletin de la Société préhistorique française, 80 , p. 98-99.

TIXIER J. (1963) - Typologie de l'Épipaléolithique du Maghreb, II, Paris, Arts et métiers graphiques (Mémoires du CRAPE 2), 210 p.

Laure-Amélie LELOUVIER

INRAP et UMR 5608 du CNRS «TRACES »

Centre archéologique de Toulouse-Saint-Orens

ZA des Champs-Pinsons

13, rue du Négoce, 31650 Saint-Orens-de-Gameville laure-amelie.lelouvier@inrap.fr

Bruno BOSC-ZANARDO

Doctorant UMR 5608 du CNRS «TRACES » Université Toulouse - Le Mirail

Maison de la recherche

5, allée Antonio-Machado, 31000 Toulouse cedex bruno.bosc-zanardo@club-internet.fr

Laurent BRUXELLES

INRAP et UMR $5608 \mathrm{du}$ CNRS «TRACES », GAES, university of Witwatersrand, Johannesburg Centre archéologique Méditerranée 561, rue Étienne-Lenoir KM delta, 30900 Nîmes laurent.bruxelles@inrap.fr

Pierre CHALARD

SRA de Midi-Pyrénées

et UMR 5608 du CNRS «TRACES»

Hôtel des Chevaliers de Saint-Jean

32, rue de la Dalbade, 31080 Toulouse cedex 6 pierre.chalard.biberson@culture.gouv.fr

Marc JARRY

INRAP et UMR 5608 du CNRS «TRACES » Centre archéologique de Toulouse-Saint-Orens

ZA des Champs-Pinsons

13, rue du Négoce, 31650 Saint-Orens-de-Gameville marc.jarry@inrap.fr 